\title{
Clinical score and rapid antigen detection test to guide antibiotic use for sore throats: randomised controlled trial of PRISM (primary care streptococcal management)
}

\author{
(c) (1) (9) OPEN ACCESS
}

The authors of this article (BMJ 2013;347:f5806, doi:10.1136/ bmj.f5806) have discovered minor errors in the reported complication rates. The article stated that no patients had suppurative complications. In fact, four patients in the first phase of the trial (one in the delayed antibiotic group and three in the clinical score group) and three in the FeverPAIN phase (one in the delayed antibiotic group and two in the antigen test group) had these complications. 